\title{
Asymmetrical methyltransferase PRMT3 regulates human mesenchymal stem cell osteogenesis via miR-3648
}

\author{
Zhang Min ${ }^{1,2}$, Liu Xiaomeng ${ }^{3}$, Li Zheng ${ }^{1,2}$, Du Yangge ${ }^{1,2}$, Liu Xuejiao ${ }^{1,2}$, Lv Longwei ${ }^{1,2}$, Zhang Xiao ${ }^{1,2}$, Liu Yunsong ${ }^{1,2}$, \\ Zhang Ping ${ }^{1,2}$ and Zhou Yongsheng (10)
}

\begin{abstract}
Histone arginine methylation, which is catalyzed by protein arginine methyltransferases (PRMTs), plays a key regulatory role in various biological processes. Several PRMTs are involved in skeletal development; however, their role in the osteogenic differentiation of mesenchymal stem cells (MSCs) is not completely clear. In this study, we aimed to elucidate the function of PRMT3, a type-I PRMT that catalyzes the formation of $\omega$-mono- or asymmetric dimethyl arginine, in MSCs osteogenesis. We found that PRMT3 promoted MSCs osteogenic commitment and bone remodeling. PRMT3 activated the expression of miR-3648 by enhancing histone $\mathrm{H} 4$ arginine 3 asymmetric dimethylation (H4R3me2a) levels at promoter region of the gene. Overexpression of miR-3648 rescued impaired osteogenesis in PRMT3-deficient cells. Moreover, administration of Prmt3 shRNA or a chemical inhibitor of PRMT3 (SGC707) caused an osteopenia phenotype in mice. These results indicate that PRMT3 is a potential therapeutic target for the treatment of bone regeneration and osteopenia disorders.
\end{abstract}

\section{Introduction}

Bone is a dynamic organ that undergoes persistent remodeling over the course of life. Bone homeostasis and remodeling mainly involves bone destruction executed by osteoclasts, and bone deposition mediated by osteoblasts derived from mesenchymal stem cells $(\mathrm{MSCs})^{1,2}$. This complex process requires balance and synchronized regulation at different control levels. MSCs are multipotent cells with the ability to develop into various cell types, such as osteoblasts, adipocytes, and chondrocytes under defined conditions ${ }^{3}$. Diminished MSC differentiation

Correspondence: Zhang Ping (zhangping332@bjmu.edu.cn) or Zhou Yongsheng (kqzhouysh@hsc.pku.edu.cn)

${ }^{1}$ Department of Prosthodontics, Peking University School and Hospital of Stomatology, 100081 Beijing, China

${ }^{2}$ National Engineering Lab for Digital and Material Technology of Stomatology, National Clinical Research Center for Oral Diseases, Peking University School and Hospital of Stomatology, 100081 Beijing, China

Full list of author information is available at the end of the article.

These authors contributed equally: Zhang Min, Liu Xiaomeng

Edited by $Y$. Shi contributes to the etiology of osteopenic diseases associated with aging, post-menopause, and diabetes mellitus $^{4-6}$. MSCs are a promising prospect for regenerative medicine, especially for bone repair ${ }^{7-9}$. The mechanism underlying osteogenesis, however, remains largely unknown. This is an impediment to the clinical application of MSCs. Understanding the molecular mechanisms of MSC lineage commitment is therefore essential for developing novel therapies for diseases involving reduced MSCs function.

Arginine methylation is a widespread post-translational modification, and is as common as ubiquitination and phosphorylation. Protein methylation mediated by protein arginine methyltransferases (PRMTs) influences various biological processes, including RNA and protein subcellular localization, transduction of signals, RNA splicing, transcription and chromatin remodeling, and DNA repair. PRMTs, act on a wide range of substrates, including histones and non-histones ${ }^{10-13}$. PRMTs play important roles in cell fate determination of blood progenitors and

\section{(c) The Author(s) 2019}

(c) (i) Open Access This article is licensed under a Creative Commons Attribution 4.0 International License, which permits use, sharing, adaptation, distribution and reproduction cc) in any medium or format, as long as you give appropriate credit to the original author(s) and the source, provide a link to the Creative Commons license, and indicate if changes were made. The images or other third party material in this article are included in the article's Creative Commons license, unless indicated otherwise in a credit line to the material. If material is not included in the article's Creative Commons license and your intended use is not permitted by statutory regulation or exceeds the permitted use, you will need to obtain permission directly from the copyright holder. To view a copy of this license, visit http://creativecommons.org/licenses/by/4.0/. 
diverse metabolic processes associated with human diseases $^{14-18}$. PRMTs play critical roles in skeletal development and bone homeostasis. PRMT1 modulates craniofacial bone formation as an upstream regulator of $M s x 1^{19}$. PRMT1 is an indispensable regulator of RANKLinduced osteoclast differentiation. PRMT1 activated osteoclast function and led to bone loss in OVX mice ${ }^{20}$. PRMT5 activates RANKL-induced osteoclast differentiation, which involves the regulation of CXCL10 and RSAD $2^{21}$. Pharmacological inhibition of Prmt5 in the ST2 and W-20 cell lines inhibited colony-forming units and promoted osteogenesis ${ }^{22}$. Thus, arginine methylation is essential for the tight regulation of cell fate decision during embryonic development and homeostasis maintenance.

PRMT3 is a type-I PRMT that catalyzes the formation of $\omega$-mono- or asymmetric dimethyl arginine, and has a unique substrate-binding $\mathrm{C}_{2} \mathrm{H}_{2} \mathrm{Zn}$ finger domain at its $\mathrm{N}$ terminus and a catalytic domain at its $\mathrm{C}$-terminus ${ }^{23,24}$. RpS2 was first reported as the substrate of PRMT3 in the cytoplasm $^{25,26}$. Prmt 3 knock-out cell lines exhibited decreased rpS2 methylation levels ${ }^{27}$. PRMT3 also modulates dendritic spine maturation in rats by interaction with $\mathrm{rpS} 2^{28}$. PRMT3 was related to hepatic lipogenesis as a cofactor to LXR $\alpha$ in the nucleus. High expression levels of PRMT3 are associated with non-alcoholic fatty liver disease (NAFLD) ${ }^{29}$. PRMT3 methylates PABPN1 and is involved in oculopharyngeal muscular dystrophy, resulting from polyalanine expansion in PABPN $1^{30,31}$. DAL-1/ 4.1B, a tumor suppressor, interacts with PRMT3 and suppresses its methyltransferase activity, indicating a potential role of PRMT3 in control of tumor growth ${ }^{32,33}$. In patients with atherosclerosis, PRMT3 expression was increased in myocardial tissues, along with downregulation of genes that promotes NO synthesis ${ }^{34}$. However, the role of PRMT3 in skeletal development has not been studied. Prmt3-null mice exhibited delayed embryonic development; the embryos were significantly smaller than those of wild-type mice, but could survive and achieve normal size during adulthood ${ }^{27}$. We aimed to investigate the potential effects of PRMT3 on osteoblastic commitment of MSCs and maintenance of bone homeostasis.

In the current study, we observed that PRMT3 is an essential regulator of MSC-mediated osteogenesis and bone homeostasis. Importantly, PRMT3 promoted the osteogenic differentiation of MSCs by increasing H4R3me2a levels in the DNA regions $343 \mathrm{bp}$ upstream and $505 \mathrm{bp}$ downstream of the transcription starting site (TSS) of miR-3648, which were likely promoter regions. PRMT3 deficiency contributed to bone loss in mice. Our findings uncovered a potential mechanism by which PRMT3 affects osteogenic differentiation of MSCs, and suggested that targeting PRMT3 might be an effective therapeutic strategy to treat bone metabolic disease and in bone regenerative medicine.

\section{Results \\ PRMT3 and H4R3me2a could be involved in MSCs osteogenesis}

To investigate the potential role of PRMT3 in osteoblastic commitment of MSCs, the expression of PRMT3 in human MSCs was detected after osteoinductive culture. As shown in Fig. 1a-c, qRT-PCR and western blotting analysis revealed that PRMT3 was strongly induced, along with the upregulation of the osteogenic marker RUNX2. In addition, we found that histone $\mathrm{H} 4$ dimethyl Arg3 asymmetric methylation (H4R3me2a) catalyzed by PRMT3 was upregulated after osteogenic induction (Fig. 1d). To assess the potential role of PRMT3 in bone homeostasis, we created ovariectomized (OVX) model mice. We then determined whether the expression level of PRMT3 was altered in impaired MSCs. Microcomputed tomography (micro-CT) analysis and hematoxylin and eosin (H\&E) staining exhibited that the trabecular area in femurs were significantly reduced in the OVX group compared to those in the SHAM group at 6 (Fig. 1e and Supplementary Fig. 1a) and 12 weeks after operation (Fig. $1 \mathrm{~g}$ and Supplementary Fig. 1b). Next, BMMSCs were isolated from mouse bone marrow by the traditional flush method. As shown in Supplementary Fig. 1c, d, BMMSCs from OVX mice showed impaired osteogenic ability based on ALP staining and quantification. We then checked the expression of PRMT3 and H4R3me2a in BMMSCs derived from OVX mice. As shown in Fig. 1f, h, PRMT3 and H4R3me2a expression levels were significantly decreased in BMMSCs from OVX mice, which were consistently correlated with the RUNX2 levels.

\section{PRMT3 arginine methyltransferase activity is required for the promotion of osteoblastic differentiation of MSCs}

To explore the role of PRMT3 in MSCs fate determination, we constructed stable PRMT3 knockdown MSCs by using lentiviruses expressing PRMT3 shRNA. Two shRNA sequences against PRMT3 were designed to exclude off-target effects. The majority of infected cells were GFP-positive (Supplementary Fig. 2a). The knockdown efficiency of PRMT3 was evaluated by qRT-PCR (Supplementary Fig. 2b) and western blotting (Fig. 2a). After treating MSCs with osteoinductive media for 1 week, ALP activity was greatly suppressed by knockdown of PRMT3 (Fig. 2b, c). In addition, alizarin red S staining and quantification indicated reduced calcium deposition in PRMT3 knockdown cells 14 days after osteogenic induction (Fig. 2d, e). Furthermore, knockdown of PRMT3 decreased the expression levels of the osteoblastic differentiation indicators $R U N X 2$ and $O C N$ (Fig. 2f, g). Subsequently, we determined whether the 


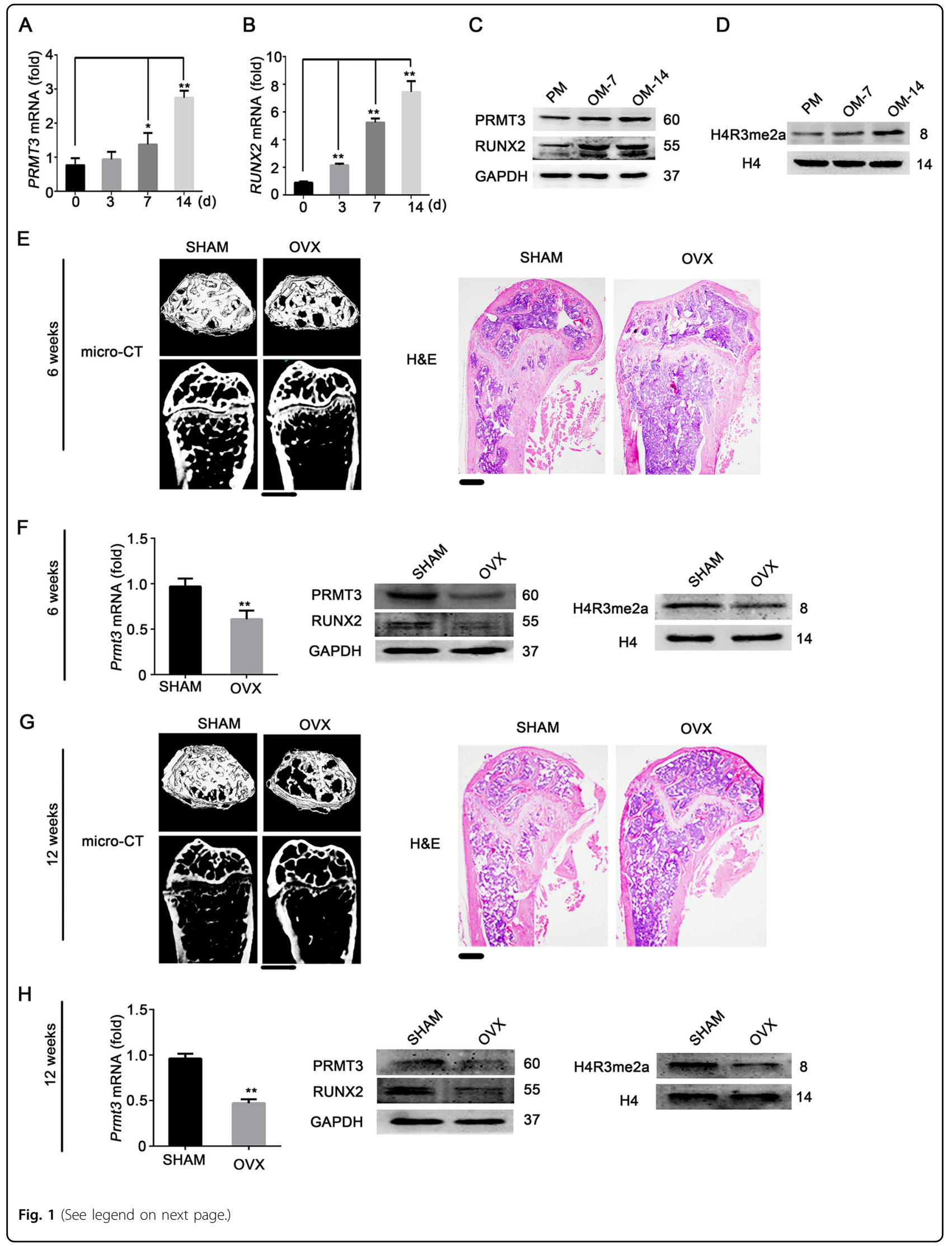


(see figure on previous page)

Fig. 1 PRMT3 and H4R3me2a are likely involved in osteogenesis. a, b Expression profiles of PRMT3 (a) and RUNX2 (b) during hMSCS osteogenic differentiation, determined by qRT-PCR analysis. Data are shown as mean \pm SD; $n=3$ independent experiments; *: compared with day $0, P<0.05$; **: compared with day $0, P<0.01$ by Student's $t$-tests. $\mathbf{c}$ Western blotting analysis of PRMT3 and RUNX2 protein levels during osteoblastic differentiation of hMSCs. GAPDH was used as an internal control. PM, proliferation medium; OM, osteogenic medium. $\mathbf{d}$ Western blotting analysis of dimethylasymmetric methylation level of H4R3 during osteogenic differentiation of hMSCs. H4 was used as an internal control. e Micro-CT image and H\&E staining of OVX mice at 6 weeks. Scale bar for micro-CT image: 1 mm; scale bar for H\&E staining: $200 \mu \mathrm{m}$. $\mathbf{f}$ qRT-PCR analysis of Prmt 3 mRNA level (left), western blotting analysis of PRMT3 and RUNX2 protein levels (middle), and H4R3me2a (right) in BMMSCs of OVX mice at 6 weeks. Data are shown as mean \pm SD; $n=3$ independent experiments; ${ }^{* *} P<0.01$ compared with SHAM group by Student's $t$-tests. $\mathbf{g}$ Micro-CT image and H\&E staining of OVX mice at 12 weeks. Scale bar for micro-CT image: $1 \mathrm{~mm}$; scale bar for H\&E staining: $200 \mu \mathrm{m}$. $\mathbf{h}$ qRT-PCR analysis of Prmt3 mRNA level (left), western blotting analysis of PRMT3 and RUNX2 protein levels (middle), and H4R3me2a (right) in BMMSCs of OVX mice at 12 weeks. Data are shown as mean \pm $\mathrm{SD} ; n=3$ independent experiments; ${ }^{* *} P<0.01$ compared with SHAM group by Student's $t$-tests

arginine methyltransferase activity of PRMT3 was involved in the modulation of osteoblastic commitment. The H4R3me2a expression levels were reduced in PRMT3 knockdown cells (Fig. 2h). In addition, a catalytically dead mutant of PRMT3 (deletion of 37 amino acid in Cterminal) was generated (Fig. 2i). We also constructed a PRMT3 rescue cell line by transfecting the vector, FlagPRMT3 (wild-type, WT), and Flag-PRMT3 mutant (Mut) in MSCs/PRMT3sh (Supplementary Fig. 2c). Wild-type PRMT3 could rescue the expression of H4R3me2a in PRMT3sh cells, while mutant PRMT3 could not (Fig. 2j). Moreover, only wild-type PRMT3 but not mutant PRMT3 could promote osteoblastic differentiation, as shown by ALP staining and quantification (Fig. $2 \mathrm{k}, \mathrm{l}$ ). Thus, our data suggested that PRMT3 could promote the osteogenic differentiation of MSCs through its arginine methyltransferase activity.

\section{PRMT3 is a positive regulator of MSC-mediated bone formation in vivo}

To confirm the effect of PRMT3 in the osteoblastic differentiation of MSCs, we mixed the MSCs/PRMT3sh with beta-tricalcium phosphate $(\beta-\mathrm{TCP})$ particles, and implanted the hybrids into the subcutaneous tissue of nude mice. $\mathrm{H} \& \mathrm{E}$ staining indicated that deficiency of PRMT3 led to less bonelike tissue formation compared with the controls (Fig. 3a). Consistent with this, Masson's trichrome staining displayed less collagen deposition (Fig. 3b). Immunohistochemical (IHC) staining indicated less positive staining for osteogenic marker OCN was found in the PRMT3sh group (Fig. 3c). Next, we performed the same experiments with the PRMT3 rescue cell line. As shown in Fig. 3d-f, only wild-type PRMT3 could elevate the osteogenic capacity of PRMT3sh cells in vivo, which corroborated the in vitro data. These data suggested that the arginine methyltransferase activity of PRMT3 was necessary for the modulation of osteoblastic differentiation.

\section{PRMT3 modulates the osteoblastic differentiation of MSCs by targeting miR-3648}

In order to elucidate the key mechanisms underlying PRMT3-mediated modulation of MSCs osteogenesis, we performed ChIP-seq to determine the potential targets of PRMT3 and H4R3me2a. As shown in Table 1A, B, 17 PRMT3 and 29 H4R3me2a enriched regions were found, wherein miR-3648 was determined as the co-binding site of PRMT3 and H4R3me2a (Fig. 4a). ChIP-qPCR results showed that PRMT3 knockdown decreased H4R3me2a levels in the promoter region of miR-3648 (Fig. 4b). To confirm whether miR-3648 expression is directly regulated by PRMT3, we examined miR-3648 expression in PRMT3 knockdown cells. As shown in Fig. 4c, miR-3648 was significantly inhibited by PRMT3 deficiency. We also detected miR-3648 in the PRMT3 rescue cell line, and found that forced expression of wild-type but not mutant PRMT3 increased the expression level of miR-3648 (Fig. 4d). To explore the function of miR-3648 in osteogenic differentiation, we treated MSCs with miR-3648 mimic or inhibitor-the overexpression or knockdown efficiency is shown in Supplementary Fig. 3a-b. Overexpression of miR-3648 enhanced ALP activity (Fig. 4e, f) and calcium deposition (Fig. 4g, h) in MSCs treated with osteogenic inducer. The mRNA expression levels of RUNX2 and $O C N$ were also increased in the miR-3648 treated group (Fig. 4i, j). In addition, MSCs treated with miR-3648 inhibitor showed reduced ALP activity (Supplementary Fig. 3c-d) and calcium deposition (Supplementary Fig. 3e-f). Furthermore, we overexpressed miR-3648 in PRMT3 knockdown cells to analyze whether miR-3648 could rescue impaired osteogenic capacity. ARS staining and quantification revealed that overexpression of miR3648 significantly elevated mineralized nodule formation in PRMT3 knockdown cells (Fig. 4k, l). Collectively, our data suggest that PRMT3 promotes osteogenesis by modulating the expression of miR-3648.

\section{Lentivirus-mediated PRMT3 downregulation leads to osteopenia phenotype in mice}

Since PRMT3 plays a key role in the osteogenic differentiation of MSCs, we determined the effect of lentivirusmediated PRMT3 downregulation on bone homeostasis in mice. Prmt3 shRNA or negative control (NC) groups were injected through tail vein. Micro-CT analysis showed that Prmt3 shRNA treatment reduced the number 


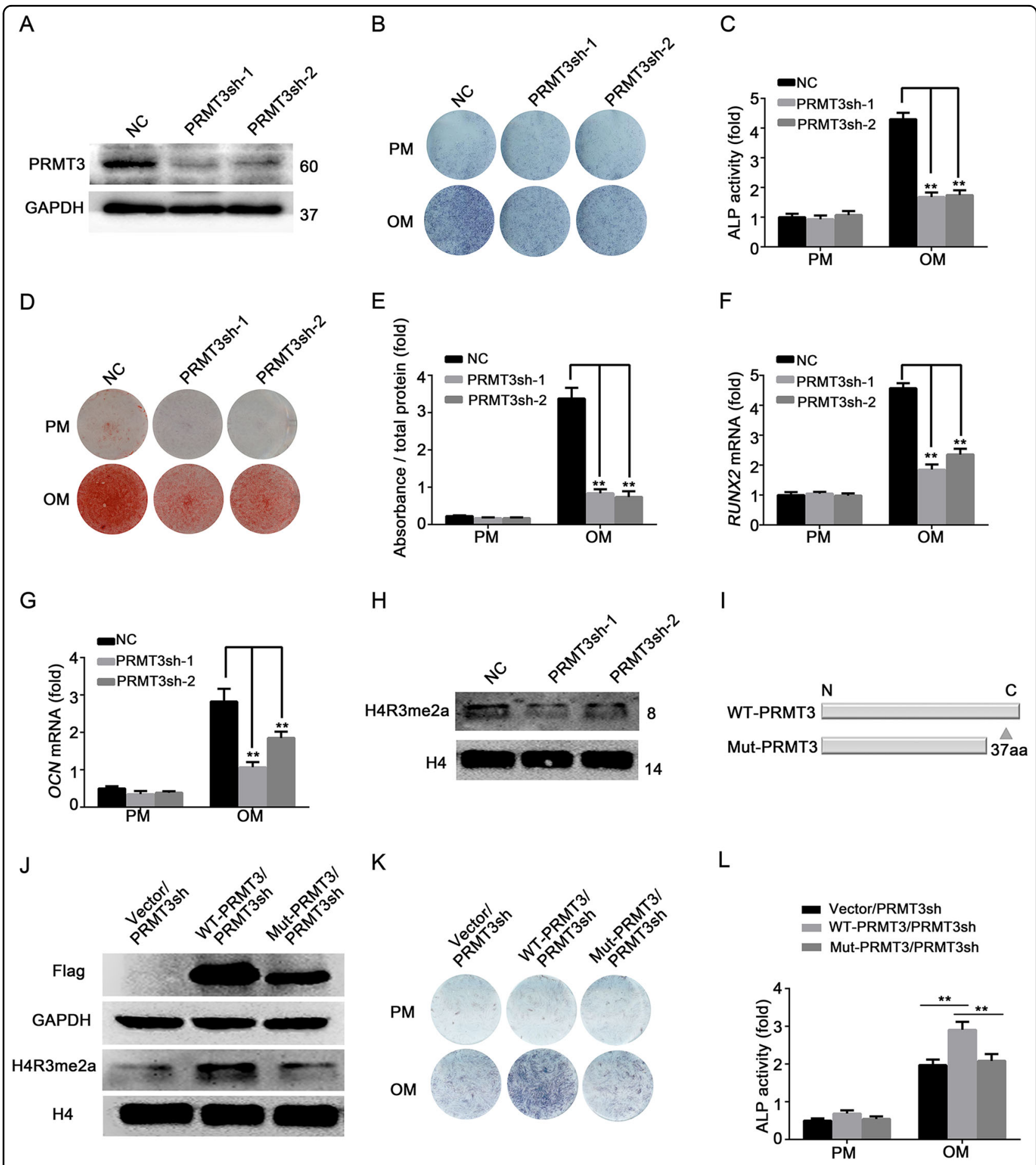

Fig. 2 PRMT3 arginine methyltransferase activity is essential for the PRMT3-mediated promotion of MSCs osteogenic differentiation.

a Western blotting analysis of PRMT3 knockdown efficiency; GAPDH was used as internal control. b, c ALP activity was significantly suppressed by knockdown of PRMT3 at 7 days after osteogenic induction, as indicated by ALP staining (b) and quantification (c). Data are shown as mean \pm SD; $n=$ 3 independent experiments; ${ }^{*} P<0.01$ compared with NC-OM by Student's $t$-tests. d, e PRMT3 knockdown exhibited decreased extracellular matrix mineralization in cells at 14 days after osteogenic induction, as shown by Alizarin Red S staining (d) and calcium quantitative analysis (e). Data are shown as mean \pm SD; $n=3$ independent experiments; ${ }^{* *} P<0.01$ compared with NC-OM by Student's $t$-tests. $\mathbf{f}, \mathbf{g}$ Knockdown of PRMT3 inhibited the expressions of RUNX2 (f) and OCN $(\mathbf{g})$ as determined by qRT-PCR. Data are shown as mean \pm SD; $n=3$ independent experiments; ${ }^{* *} P<0.01$ compared with NC-OM by Student's t-tests. $\mathbf{h}$ Western blotting analysis of H4R3me2a expression level in PRMT3 knockdown cells. H4 was used as an internal control. i Schematic illustration of catalytic dead mutant PRMT3. j Western blotting analysis of H4R3me2a expression level with forced expression of wild-type or mutant PRMT3 in PRMT3sh cells. H4 was used as an internal control. $\mathbf{k}$, I ALP staining (k) and quantification (I) of PRMT3 rescue cell lines after 7-day osteogenic differentiation. Data are shown as mean $\pm S D ; n=3$ independent experiments; ${ }^{* *} P<0.01$ by Student's $t$-tests 

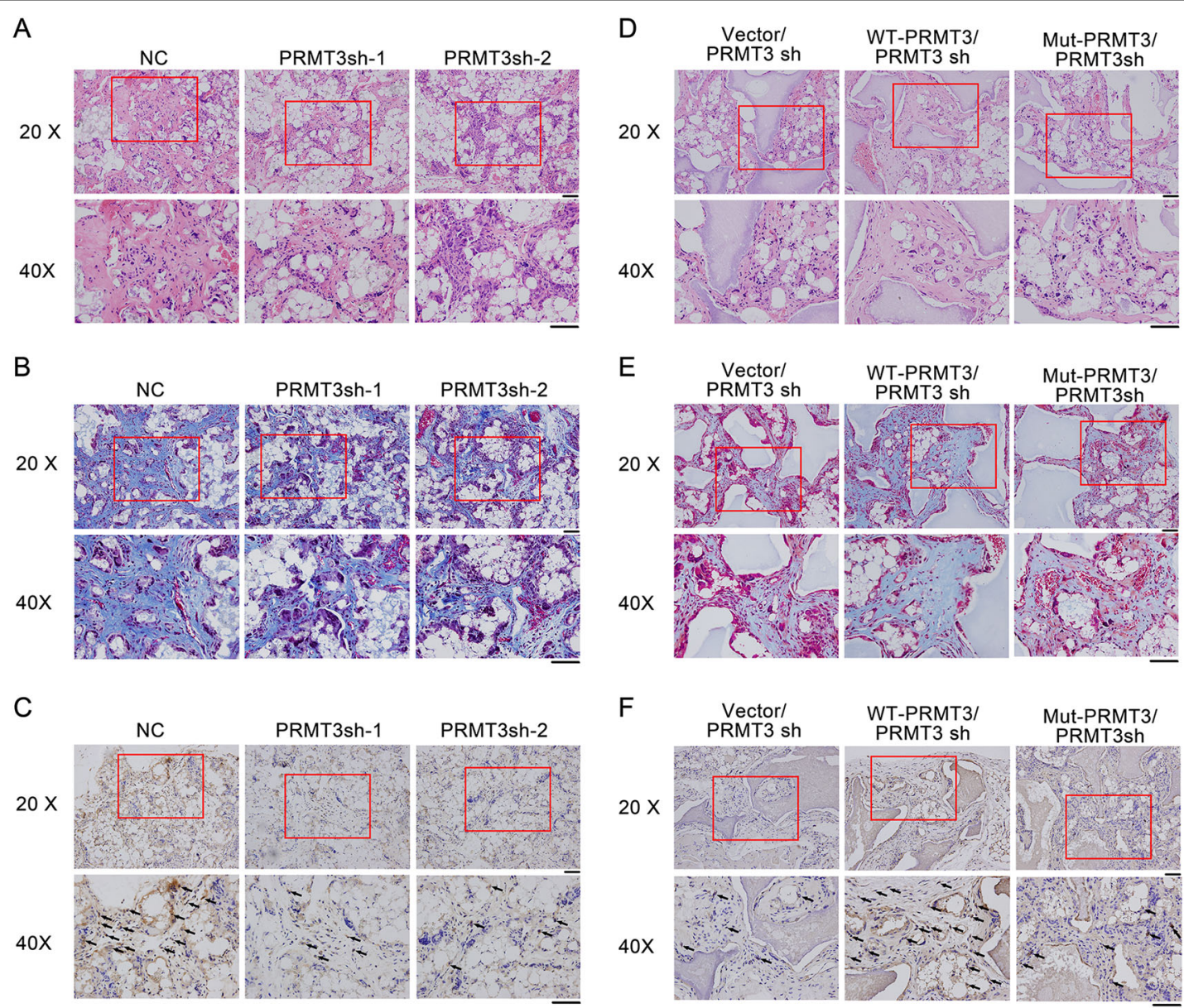

Fig. 3 PRMT3 is a positive regulator of MSC-mediated bone formation in vivo. a H\&E staining of PRMT3 knockdown cells. $\mathbf{b}$ Masson's trichrome staining of PRMT3 knockdown cells. $\mathbf{c}$ HC staining for OCN in PRMT3 knockdown cells. $\mathbf{d}$ H\&E staining of PRMT3 rescue cells. e Masson trichrome staining of PRMT3 rescue cells. $\mathbf{f} \mathrm{HC}$ staining for OCN in PRMT3 rescue cells. H\&E staining, hematoxylin-eosin staining; IHC staining, immunohistochemistry staining; Scale bars represents $50 \mu$ m; Black arrows indicate positive staining of OCN; $n=6$

and thickness of trabecular bones (Fig. 5a). Bone histomorphometry analysis showed that the values of bone mineral density (BMD), bone volume/tissue volume (BV/ $\mathrm{TV}$ ), and trabecular thickness (Tb.Th) were reduced following Prmt3 shRNA injection, and the trabecular spacing (Tb.Sp) was increased (Fig. 5b). To validate the micro-CT evaluation, histological analyses of bone tissue slices were performed. H\&E staining showed that the trabecular bone volume of Prmt 3 shRNA mice was dramatically reduced in the distal femur compared with that in the NC group (Fig. 5c). The expression levels of PRMT3 and the osteogenesis markers RUNX2 and OCN were reduced in the compact bone and bone marrow in Prmt 3 shRNA mice, as indicated by immunohistochemical analyses (Fig. 5d). IHC staining of ALP also exhibited less positive marker in Prmt3 shRNA mice (Supplementary Fig. 4a). Osteogenic differentiation and osteoclast differentiation are coupled in vivo. Tartrate-resistant acid phosphatase (TRAP) staining showed that treatment with Prmt3 shRNA elevated the osteoclast number in mice (Supplementary Fig. 4b).

\section{The PRMT3 inhibitor SGC707 inhibits osteogenic differentiation of MSCs}

To confirm our findings, we explored the effect of the selective PRMT3 inhibitor SGC707 on the osteogenic differentiation of MSCs. SGC707 binds to the allosteric site of PRMT3 and inhibits its methyltransferase activity in cells ${ }^{35}$. CCK8 assay showed that up to $50 \mu \mathrm{M}$ SGC707 had no significant effect on the proliferation ability of MSCs (Supplementary Fig. 5a). Moreover, SGC707 significantly inhibited the expression of H4R3me2a at concentrations $>10 \mu \mathrm{M}$ (Supplementary Fig. 5b). MSCs were cultured in osteogenic medium following treatment with various concentrations of SGC707. ALP staining and quantification indicated that SGC707 treatment attenuated ALP activity in MSCs (Fig. 6a, b). Similarly, ARS staining and quantification indicated 
Table 1A PRMT3 enriched regions obtained by peak calling

\begin{tabular}{|c|c|c|c|c|c|c|}
\hline Chr & Start & End & Length & Abs summit & Pileup & Fold enrichment \\
\hline chr1 & 91852810 & 91853102 & 293 & 91852969 & 106.94 & 26.98379 \\
\hline chr1 & 156186298 & 156186560 & 263 & 156186431 & 93.4 & 9.44046 \\
\hline chr10 & 42389330 & 42389534 & 205 & 42389480 & 117.41 & 2.19279 \\
\hline chr11 & 85195028 & 85195224 & 197 & 85195101 & 45.39 & 11.59822 \\
\hline chr12 & 49578773 & 49578862 & 90 & 49578799 & 10.91 & 8.81699 \\
\hline chr12 & 127650485 & 127650648 & 164 & 127650560 & 21.82 & 11.41175 \\
\hline chr16 & 33963053 & 33963318 & 266 & 33963121 & 44.52 & 9.10399 \\
\hline chr16 & 33963887 & 33964033 & 147 & 33963968 & 51.5 & 27.6334 \\
\hline chr17 & 17286832 & 17286967 & 136 & 17286914 & 14.84 & 9.99368 \\
\hline chr19 & 27732021 & 27732268 & 248 & 27732234 & 93.4 & 2.55148 \\
\hline chr2 & 133025749 & 133025850 & 102 & 133025826 & 24.88 & 8.62626 \\
\hline chr21 & 9825493 & 9826367 & 875 & 9826191 & 78.56 & 7.95646 \\
\hline chr21 & 9826842 & 9827550 & 709 & 9827369 & 117.41 & 7.89403 \\
\hline chr5 & 71146767 & 71146900 & 134 & 71146850 & 24.88 & 8.62626 \\
\hline chr8 & 70602325 & 70602492 & 168 & 70602382 & 57.18 & 24.75641 \\
\hline $\operatorname{chr} x$ & 12995051 & 12995176 & 126 & 12995132 & 17.02 & 13.16459 \\
\hline $\operatorname{chr} x$ & 108297370 & 108297815 & 446 & 108297726 & 63.72 & 32.36231 \\
\hline
\end{tabular}

reduced calcium deposition in cells treated with SGC707 (Fig. 6c, d). Furthermore, SGC707 treatment downregulated the mRNA expression levels of the osteogenesis-related genes RUNX2, SP7, and ALP (Fig. 6e-g). We then confirmed the effect of SGC707 on osteogenesis by in vivo study. MSCs pre-treated with SGC707 $(50 \mu \mathrm{M})$ for $96 \mathrm{~h}$ were mixed with $\beta$-TCP scaffolds and implanted subcutaneously in nude mice. As shown in Fig. 6h, H\&E staining indicated that SGC707 treatment reduced eosinophilic bone-like tissue formation, and IHC staining of OCN indicated lower positive expression in cells treated with SGC707 (Fig. 6i).

\section{SGC707 treatment induces bone loss in mice}

We determined whether SGC707 has an effect on bone homeostasis similar to that of intravenous injection of Prmt3 shRNA. ALZET Pump Models were used for the controlled release of SGC707. Micro-CT analysis showed that the trabecular bone in femurs was decreased at 6 weeks with SGC707 delivery, whereas there was no significant difference between the vehicle and SGC707 groups at 2 and 4 weeks (Fig. 7a). Bone histomorphometry analysis showed that the values of BMD, BV/TV, trabecular number (Tb.N), and Tb.Th were reduced, and the trabecular spacing (Tb.Sp) was increased in mice treated with SGC707 for 6 weeks (Fig. 7b). There were no significant differences between the vehicle and SGC707 group at 2 and 4 weeks (Supplementary Fig. 6a-b). Moreover, H\&E staining showed similar results to micro$\mathrm{CT}$ analysis. The trabecular bone content and thickness of SGC707-treated mice were dramatically reduced in the distal femur compared with vehicle group at 6 weeks (Fig. 7c). We then evaluated the osteogenic capability of BMMSCs from mice treated with SGC707 for 6 weeks. Compared with the vehicle group, BMMSCs from SGC707-treated mice showed impaired ALP activity and less extracellular matrix mineralization (Fig. 7d, e). TRAP staining showed that treatment with SGC707 elevated the osteoclast number in mice (Supplementary Fig. 7).

\section{Discussion}

Disruption of the balance between osteoblast and osteoclast function plays a vital part in the pathogenesis of osteoporosis $^{36,37}$. OVX mice are typically used as an osteoporotic model ${ }^{38}$ to induce bone loss. Our study showed that BMMSCs separated from OVX mice exhibited lower ALP activity and mineralization. Moreover, PRMT3 expression level was increased during MSCs osteoblastic differentiation and decreased in BMMSCs from OVX mice, suggesting that PRMT3 plays a critical role in MSC-mediated osteogenesis and therefore the pathogenesis of osteoporosis. By using gain- and loss-offunction assays, we confirmed that PRMT3 enhances MSC osteoblastic differentiation in vitro and in vivo. 
Table 1B H4R3me2a enriched regions obtained by peak calling

\begin{tabular}{|c|c|c|c|c|c|c|}
\hline Chr & Start & End & Length & Abs summit & Pileup & Fold enrichment \\
\hline chr1 & 91852803 & 91853114 & 312 & 91852974 & 121.67 & 24.53474 \\
\hline chr1 & 97144579 & 97144683 & 105 & 97144638 & 16.94 & 8.96813 \\
\hline chr1 & 121483314 & 121483403 & 90 & 121483376 & 82.01 & 2.76691 \\
\hline chr1 & 121483783 & 121483880 & 98 & 121483817 & 79.33 & 2.86904 \\
\hline chr1 & 156186291 & 156186584 & 294 & 156186448 & 102.06 & 10.30633 \\
\hline chr10 & 98510372 & 98510509 & 138 & 98510420 & 22.28 & 15.11984 \\
\hline chr11 & 65266876 & 65267089 & 214 & 65266980 & 28.08 & 17.8396 \\
\hline chr11 & 82400759 & 82400853 & 95 & 82400799 & 13.37 & 9.97275 \\
\hline chr11 & 85195027 & 85195223 & 197 & 85195101 & 52.15 & 13.28647 \\
\hline chr12 & 49578714 & 49578962 & 249 & 49578796 & 22.73 & 17.56495 \\
\hline chr12 & 104659396 & 104659510 & 115 & 104659434 & 12.03 & 9.64742 \\
\hline chr12 & 118582745 & 118582867 & 123 & 118582817 & 11.59 & 8.68136 \\
\hline chr12 & 127650481 & 127650648 & 168 & 127650560 & 25.85 & 13.42504 \\
\hline chr16 & 33963047 & 33963323 & 277 & 33963122 & 57.49 & 11.69883 \\
\hline chr16 & 33963883 & 33964036 & 154 & 33963967 & 60.61 & 32.42842 \\
\hline chr17 & 17286811 & 17287010 & 200 & 17286900 & 24.07 & 15.81534 \\
\hline chr18 & 108194 & 108484 & 291 & 108238 & 109.19 & 2.68767 \\
\hline chr18 & 6462268 & 6462364 & 97 & 6462335 & 16.04 & 12.73486 \\
\hline chr19 & 27732017 & 27732275 & 259 & 27732047 & 98.94 & 3.8813 \\
\hline chr2 & 133025747 & 133025856 & 110 & 133025826 & 28.97 & 9.98997 \\
\hline chr21 & 9825481 & 9826366 & 886 & 9825624 & 94.93 & 9.59322 \\
\hline chr21 & 9826836 & 9827553 & 718 & 9827305 & 129.25 & 7.23614 \\
\hline chr5 & 71146756 & 71146894 & 139 & 71146830 & 29.42 & 15.20781 \\
\hline chr7 & 44507686 & 44507803 & 118 & 44507742 & 15.6 & 12.40187 \\
\hline chr7 & 132719500 & 132719624 & 125 & 132719536 & 10.25 & 5.62545 \\
\hline chr8 & 70602324 & 70602495 & 172 & 70602389 & 58.83 & 25.4601 \\
\hline chr9 & 79186754 & 79186872 & 119 & 79186805 & 10.25 & 8.40596 \\
\hline $\operatorname{chr} x$ & 12994994 & 12995207 & 214 & 12995103 & 36.99 & 27.57065 \\
\hline $\operatorname{chr} x$ & 108297368 & 108297812 & 445 & 108297679 & 73.98 & 37.68077 \\
\hline
\end{tabular}

Epigenetic modification is an intrinsic modulatory mechanism in cell development and differentiation ${ }^{39}$. Imbalance of epigenetic regulation leads to various pathologies, including cancer, inflammatory diseases, and metabolic disorders ${ }^{40-42}$. Histone methylation is an important form of epigenetic regulation, and is associated with chromatin structure and regulation of gene expression. Histone methylation mainly occurs on lysine and arginine residues ${ }^{43,44}$. Lysine methylation is required for the subtle control of MSC differentiation and osteogenesis $^{45-47}$. However, the involvement of histone arginine methylation in the lineage commitment of MSCs remains unclear. PRMT3 catalyzes asymmetric dimethylation of H4R3 ${ }^{23,48,49}$. We confirmed that H4R3 is a substrate of PRMT3 in MSCs. Knockdown of PRMT3 decreased the H4R3me2a level in MSCs. The expression levels of H4R3me2a in BMMSCs from OVX mice or MSCs under osteogenic conditions were consistent with those of PRMT3. Most importantly, forced expression of catalytically mutant PRMT3 failed to rescue the osteogenic capacity of PRMT3-deficient MSCs. Thus, the asymmetric dimethylation of H4R3 catalyzed by PRMT3 is likely a novel regulatory mechanism underlying the osteogenic differentiation of MSCs. 


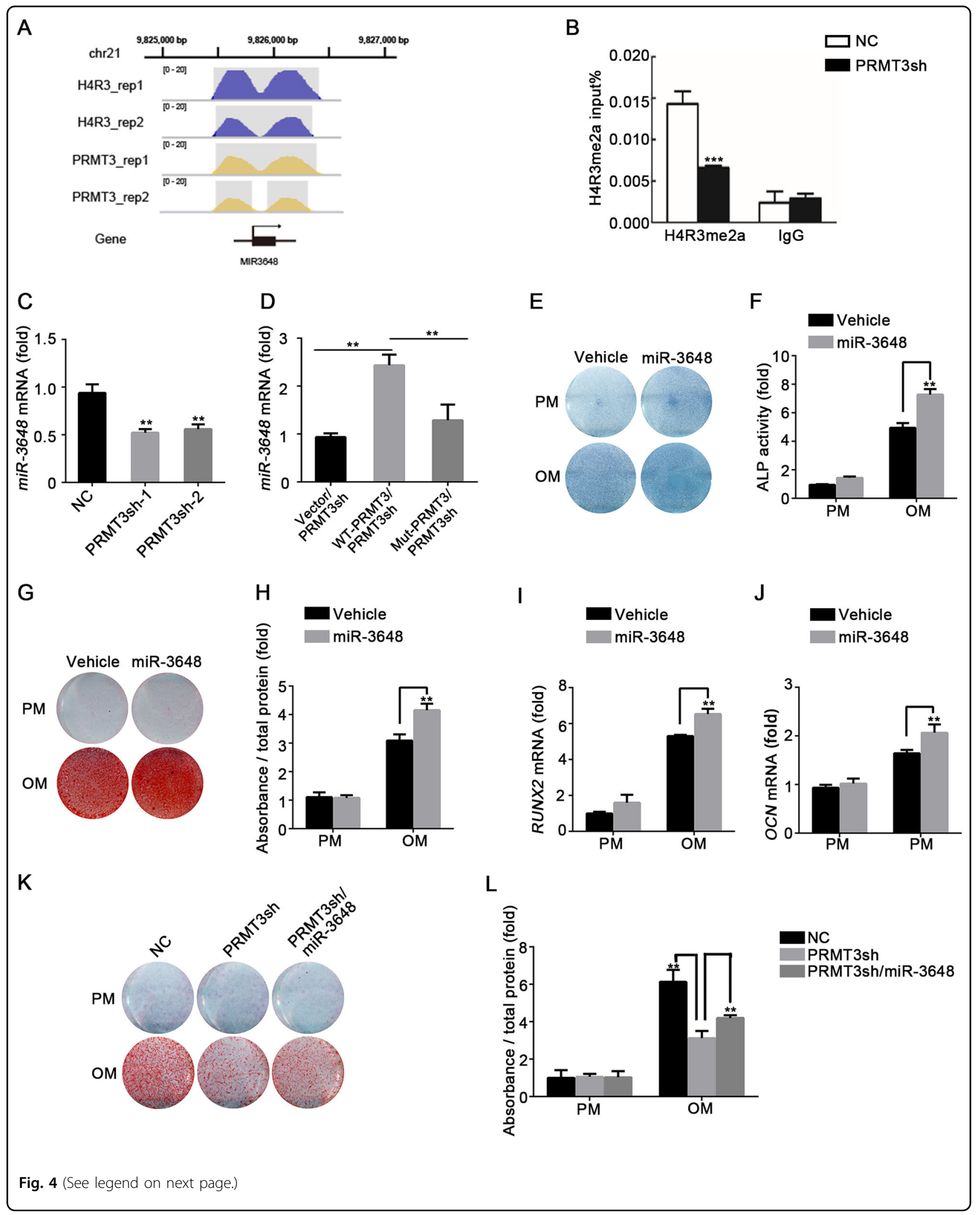


(see figure on previous page)

Fig. 4 PRMT3 modulates MSCs osteogenic differentiation by targeting miR-3648. a H4R3me2a and PRMT3 signal tracks for the loci of miR-3648. The gray shaded part indicates H4R3me2a or PRMT3 enriched region obtained by peak calling. $\mathbf{b}$ ChIP analysis showed H4R3me2a at the promoter of miR-3648 in control and PRMT3 knockdown cells. Data are shown as mean $\pm S D ; n=3$ independent experiments; *** $<0.001$ compared with NC by Student's $t$-tests. c Relative expression level of miR-3648 in PRMT3 knockdown MSCs, as determined by qRT-PCR. Data are shown as mean \pm SD; $n=3$; ${ }^{*} P<0.01$ compared with NC by Student's $t$-tests. $\mathbf{d}$ qRT-PCR analysis of miR-3648 expression in PRMT3sh MSCs with forced expression of wild-type or mutant PRMT3. Data are shown as mean \pm SD; $n=3 ;{ }^{* *} P<0.01$ by Student's $t$-tests. e, $\mathbf{f}$ ALP staining (e) and quantification (f) in miR-3648 overexpression cells on day 7 after osteogenic induction. Data are shown as mean \pm SD; $n=3 ;{ }^{* *} P<0.01$ by Student's $t$-tests. $\mathbf{g}, \mathbf{h}$ Alizarin Red $S$ staining $(\mathbf{g})$ and quantification analysis (h) in miR-3648 overexpression cells on day 14 after osteogenic induction. Data are shown as mean \pm SD; $n=$ 3; ${ }^{* *} P<0.01$ by Student's $t$-tests. $\mathbf{i}$, j Overexpression of miR-3648 promoted the expression of RUNX2 (i) on day 7 and OCN (j) on day 14 after osteogenic induction, as determined by qRT-PCR. Data are shown as mean \pm SD; $n=3$; ** $P<0.01$ by Student's $t$-tests. $\mathbf{k}$, I ARS staining (k) and quantification (I) revealed that overexpression of miR-3648 elevated the mineralized nodule formation in PRMT3 knockdown cells on day 14 after osteogenic induction. Data are shown as mean $\pm \mathrm{SD}, n=3 .{ }^{* *} P<0.01$

A

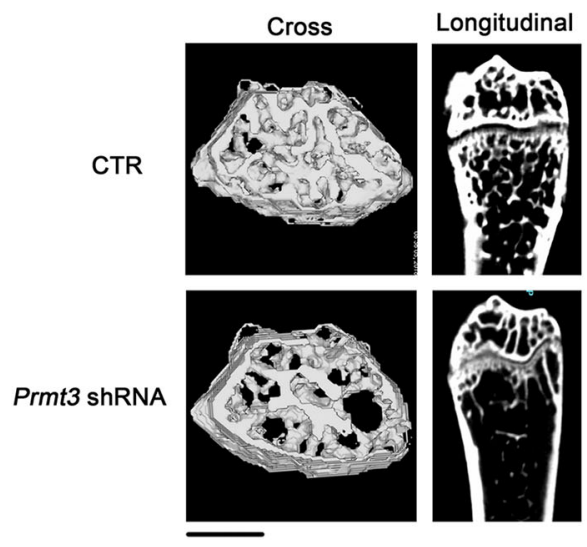

C

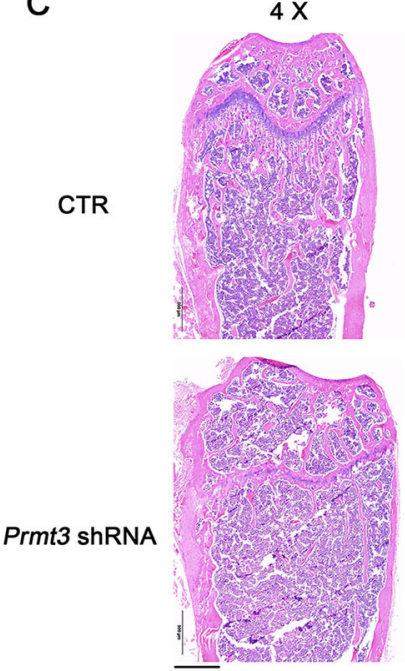

$10 x$

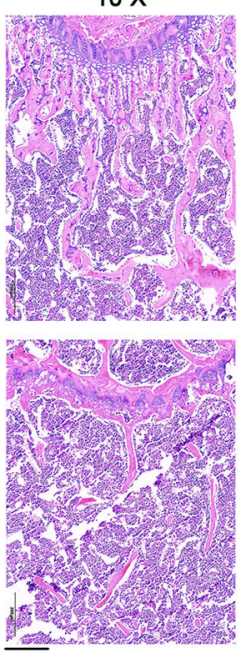

B
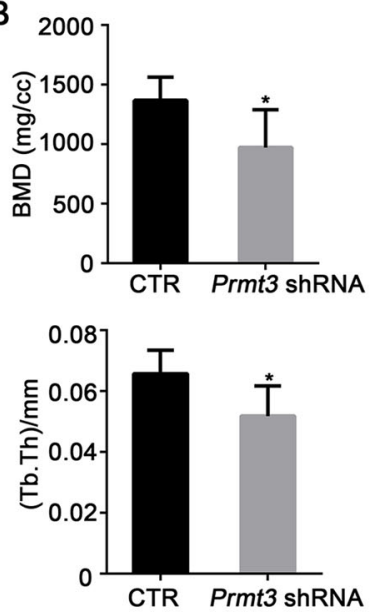

D

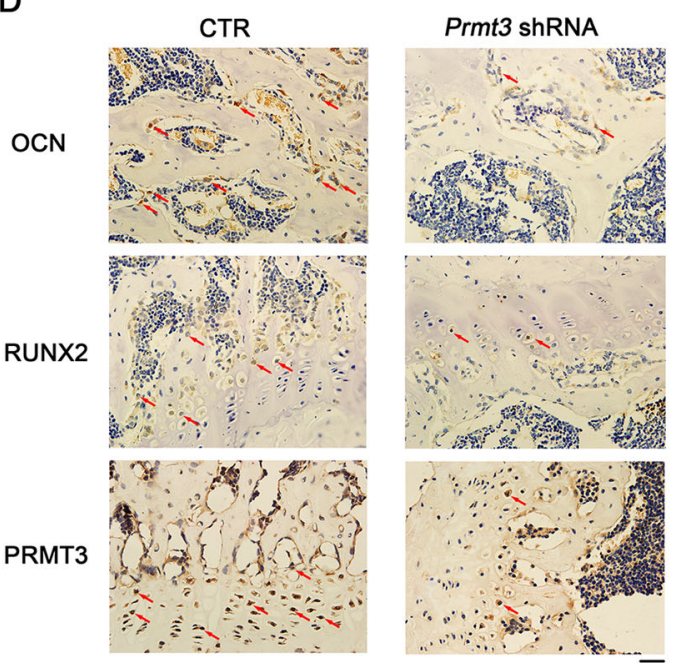

Fig. 5 Lentivirus-mediated PRMT3 downregulation leads to osteopenia phenotype in mice. a Micro-CT image of the femurs from Prmt3 shRNA treatment mice. Scale bar: $1 \mathrm{~mm}$. b Quantitative measurements of bone mineral density (BMD), bone volume/tissue volume (BV/TV), trabecular thickness (Tb.Th), and trabecular spacing (Tb.Sp). Data are shown as mean $\pm \mathrm{SD} ; n=5 ;{ }^{*} P<0.05$ by Student's $t$-tests. $\mathbf{c}$ H\&E staining of femur bone sections. Scale bar for 4X: $500 \mu \mathrm{m}$; Scale bar for 10x: $200 \mu \mathrm{m}$. d IHC staining for OCN, RUNX2, and PRMT3 of bone sections from Prmt3 shRNA treatment mice. Scale bar: $20 \mu \mathrm{m}$. Red arrows indicate positive staining 


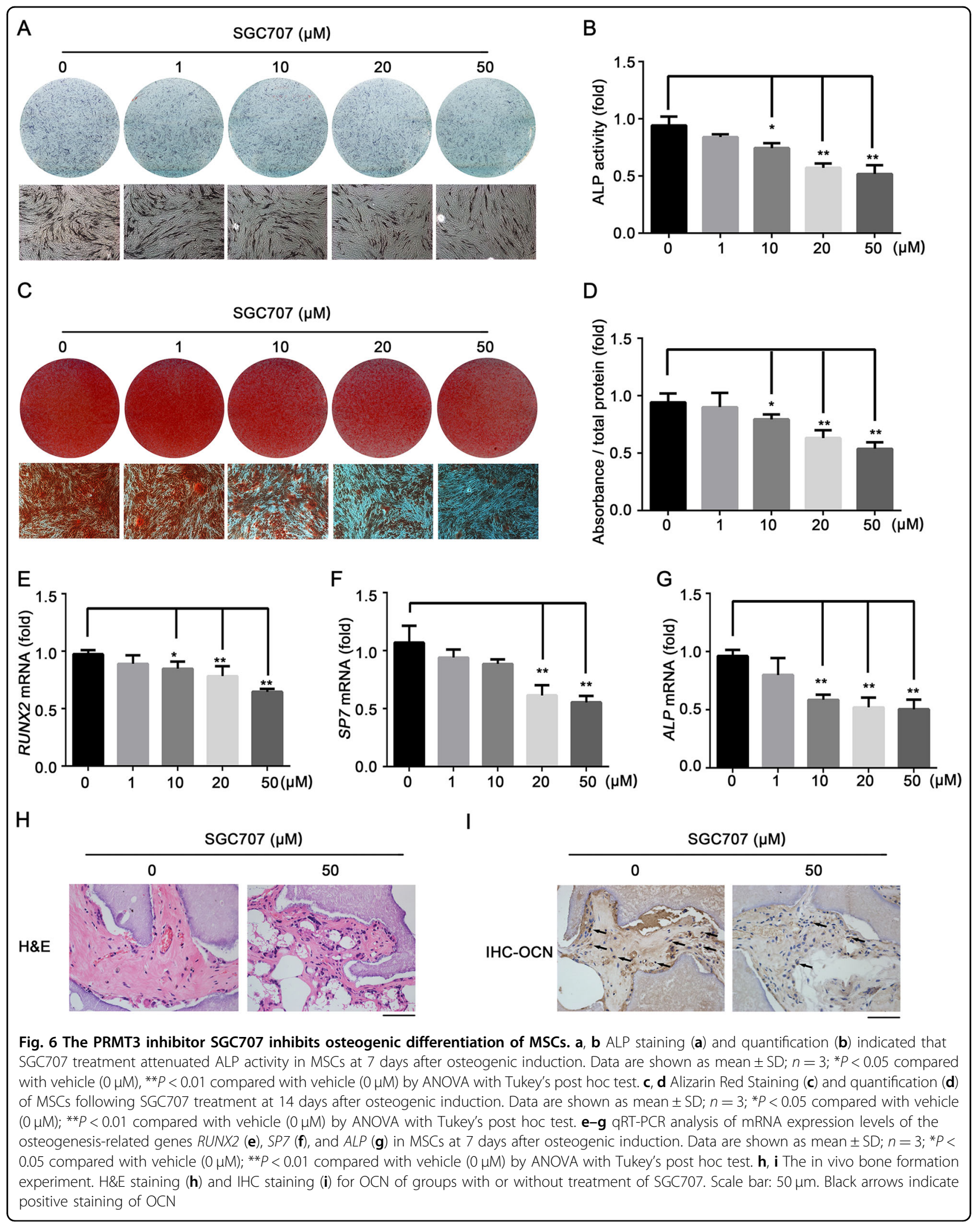



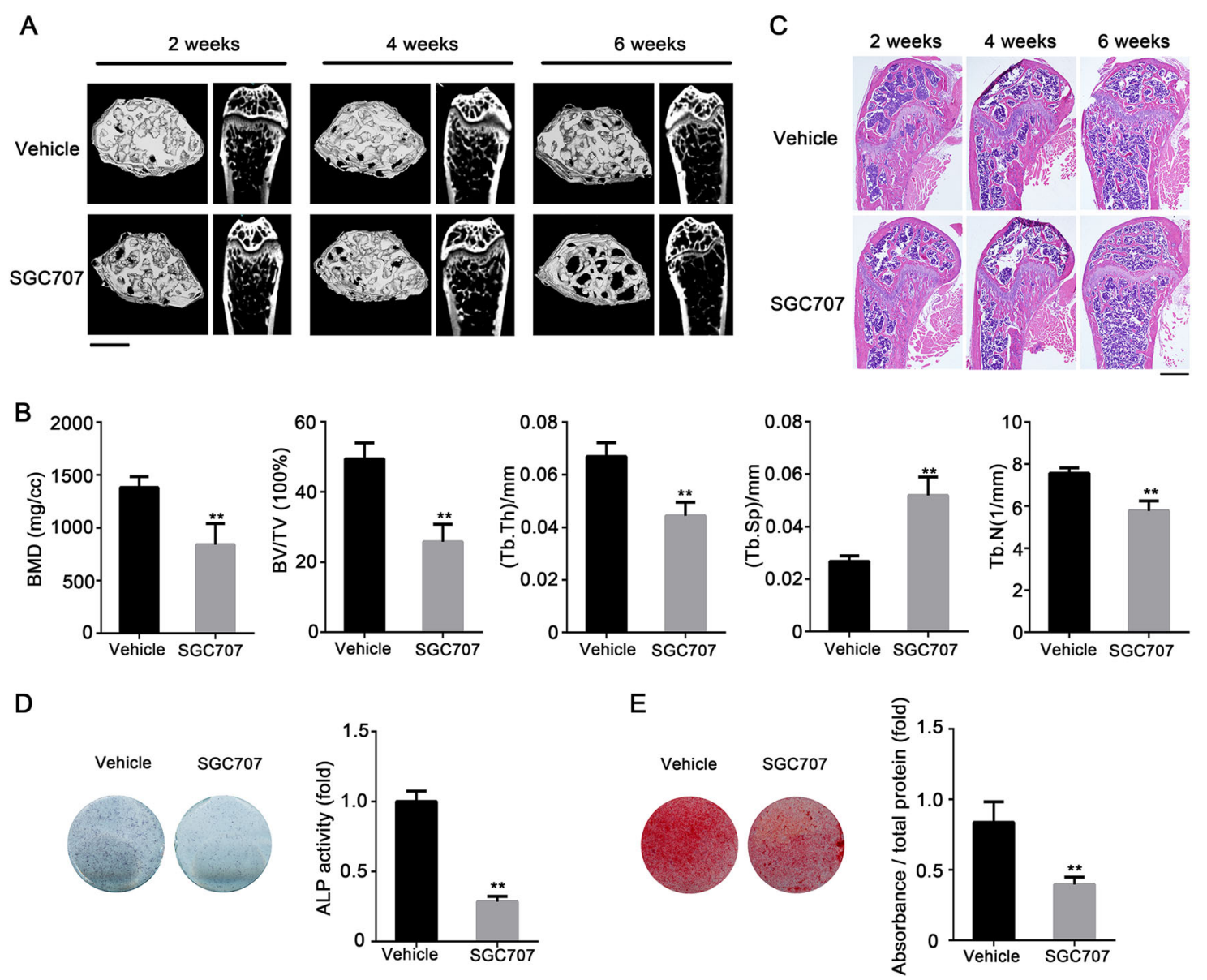

Fig. 7 SGC707 treatment induces bone loss in mice. a Micro-CT image of trabecular bone in femurs at 2, 4, and 6 weeks after administration of SGC707 or vehicle. Scale bar:1 mm. b Quantitative measurements of bone mineral density (BMD), bone volume/tissue volume (BV/TV), trabecular number (Tb.N), trabecular thickness (Tb.Th), and trabecular spacing (Tb.Sp) of femurs with SGC707 delivery for 6 weeks. Data are shown as mean \pm SD; $n=5$; ${ }^{*} P<0.01$ by Student's $t$-tests. $\mathbf{c}$ H\&E staining of trabecular bone in femurs at 2, 4, 6 weeks after SGC707 delivery. Scale bar: $500 \mu \mathrm{m}$. $\mathbf{d}$ ALP staining (left) and quantification (right) of mBMMSCs with SGC707 delivery or vehicle on day 7 after osteogenic induction. Data are shown as mean \pm SD; $n=3 ;{ }^{* *} P<0.01$ by Student's t-tests. e Alizarin Red S Staining (left) and quantification (right) of mBMMSCs following SGC707 or vehicle treatment on day 14 after osteogenic induction. Data are shown as mean \pm SD; $n=3 ;{ }^{* *} P<0.01$ by Student's $t$-tests

MicroRNAs are single-stranded RNAs of about 22 nucleotides that induce post-transcriptional repression by binding to complementary sequences and leading to mRNA degradation or repression of translation ${ }^{50}$. Substantial research has been performed to reveal the function and targets of individual miRNAs such as miR-21, miR375 , and miR-138, which are associated with osteogenic differentiation of $\mathrm{MSCs}^{51-53}$. Transcriptional or epigenetic factors are key regulators of microRNA expression. For instance, PRMT4 contributes to the expression of several myogenic-related microRNA via dimethylation of H3R17 at upstream regulatory regions ${ }^{54}$. PRMT7 epigenetically repressed the expression of miR-24-2 by upregulating H4R3me2s level in the promoter region ${ }^{55}$. The H4R3me2a marker is a modification site associated with chromatin remodeling for activation of genes ${ }^{56,57}$, including $\mathrm{pS} 2$,
IL17, and the $\beta$-globin locus ${ }^{58-60}$. Our results demonstrated that PRMT3 epigenetically increased the expression of miR-3648 by upregulating H4R3me2a. miR-3648 increases cell proliferation by direct downregulation of $\mathrm{APC}^{61}$. However, the effect of miR-3648 on osteogenesis was unclear. Our results showed that overexpression of miR-3648 promoted osteogenic differentiation, while inhibition of miR-3648 repressed osteogenic differentiation in MSCs. Moreover, forced expression of miR-3648 could rescue the osteogenic capacity of PRMT3 knockdown cells. Our results indicate that miR-3648 is a novel regulator of MSC-mediated osteogenesis. Further investigation is required to verify the direct downstream osteogenesis-related targets of miR-3648 in MSCs.

SGC707 is a first-in-class PRMT3 chemical probe that binds to the allosteric site of PRMT3 and inhibits its 
methyltransferase activity in a temporal, selective, and dose-dependent manner ${ }^{35}$. We confirmed that SGC707 could inhibit PRMT3 catalytic activity and reduce the H4R3 asymmetric dimethylation level in MSCs, with no effect on the proliferation of cells. Moreover, SGC707 suppressed the osteogenic capacity of MSCs in a dose-dependent manner, which confirmed that PRMT3 promotes osteogenic differentiation via its methyltransferase activity.

Bone homeostasis is a complex and tightly regulated process, involving the balance between new bone formation carried out by MSC-derived osteoblasts and bone resorption achieved by hematopoietic stem cell-derived osteoclasts. Factors that regulate cell proliferation and cell fate decision of both cell types may lead to osteopenia and osteoporosis $^{62}$. By systematic application of Prmt3 shRNA and the specific inhibitor SGC707, we found that PRMT3 deficiency leads to bone loss in mice. The expression levels of RUNX2, OCN and ALP were reduced in bone sections of Prmt 3 shRNA mice. BMMSCs isolated from mice treated with SGC707 for 6 weeks showed decreased osteogenic capacity. Thus, the osteopenia phenotype could be attributed to a formation-resorption imbalance, wherein diminished bone formation could not compensate for regular or excessive bone resorption. PRMTs were recently reported to participate in the RANKL-induced osteoclastogenesis $^{20,21}$. TRAP staining showed that treatment with Prmt3 shRNA or SGC707 elevated the osteoclast number in mice. Further studies are needed to explore the detailed mechanism of how PRMT3 affects the function of osteoclasts and thus regulates the process of bone resorption. Joya et al. found that SGC707 treatment impairs the hepatic lipogenesis due to administration of LXR agonists as therapeutic agents for cholesteroldriven diseases in an in vivo animal study ${ }^{49}$. Our study revealed the effect of SGC707 on bone metabolism, which should be taken into consideration for clinical application.

In conclusion, our study revealed that PRMT3 plays an essential role in the osteoblastic differentiation of MSCs by epigenetically controlling miR-3648 transcription. Prmt3 shRNA and SGC707 treatment resulted in bone reduction in mice. Our results suggested that PRMT3 is a novel regulator of bone homeostasis, and indicated that PRMT3 and H4R3me2a could be biomarkers and therapeutic targets for the diagnosis and treatment of osteoporotic diseases.

\section{Materials and methods}

\section{Cell culture and lineage differentiation}

Primary hMSCs were purchased from ScienCell Research Laboratories (Carlsbad, CA, USA). Cells at passage 3 from three independent donors were used for the assays. Proliferation media (DMEM; 10\% fetal bovine serum; $1 \%$ penicillin/streptomycin) and osteogenic media
[Proliferation media containing $100 \mathrm{nM}$ dexamethasone (Sigma-Aldrich, St. Louis, MO, USA), $0.2 \mathrm{mM}$ ascorbic acid (Sigma-Aldrich), and $10 \mathrm{mM} \beta$-glycerophosphate (Sigma-Aldrich)] were used for cell culture and osteogenic induction respectively.

\section{Isolation of primary mBMMSCs}

The bone marrow was flashed out of mouse femurs and harvested. After centrifugation for $5 \mathrm{~min}$ at $1000 \mathrm{rpm}$, the cells were collected and cultured in fresh $\alpha$-MEM mixed with $20 \%$ FBS and $1 \%$ antibiotics. Non-adherent cells were discarded at $48 \mathrm{~h}$ and attached cells were maintained until they reached full confluency. Mouse bone marrow mesenchymal stem cells (mBMMSCs) at passage 1 were used to analyze the expression levels of PRMT3 and H4R3me2a under osteoporotic conditions. Osteogenic media [fresh $\alpha$-MEM containing $100 \mathrm{nM}$ dexamethasone (Sigma-Aldrich, St. Louis, MO, USA), $0.2 \mathrm{mM}$ ascorbic acid (Sigma-Aldrich), and $10 \mathrm{mM} \beta$-glycerophosphate (Sigma-Aldrich)] was used for osteoinduction of mBMMSCs.

\section{Alkaline phosphatase staining and quantification}

Cells were cultured in 12-well plates and harvested after 7-day osteoinduction. The BCIP/NBT staining kit (CWBIO, Beijing, China) was used to for Alkaline phosphatase (ALP) staining. To determine the ALP activity, cells were rinsed three times with PBS and lysed with $1 \%$ TritonX-100 (Sigma-Aldrich) for $10 \mathrm{~min}$ on ice. The cells were then harvested and centrifuged at $12000 \mathrm{rpm}$ for $30 \mathrm{~min}$ at $4{ }^{\circ} \mathrm{C}$. BCA protein assay kits (Prod\#23225, Pierce Thermo Scientific, Waltham, MA, USA) were used to detect protein concentration. ALP activity was measured using an ALP assay kit (A059-2, Nanjing Jiancheng Bioengineering Institute, Nanjing, China). The comparison of relative ALP activity among groups was quantitatively performed after normalization to the total protein concentration.

\section{Alizarin Red $\mathrm{S}$ staining and quantification}

After osteogenic induction for 2 weeks, cells were fixed with $95 \%$ ethanol for $30 \mathrm{~min}$ at room temperature. After washing three times with distilled water, the cells were incubated with Alizarin Red S solution (2\%, pH 4.2, Sigma-Aldrich). For the quantitative detection of calcium deposition, the staining samples were incubated with $100 \mathrm{mM}$ cetylpyridinium chloride (Sigma-Aldrich) for $1 \mathrm{~h}$ and the absorbance was measured at $562 \mathrm{~nm}$ and normalized to the total protein concentration.

\section{RNA extraction, reverse transcription, and quantitative real-time PCR}

Total RNA was extracted with TRIzol reagent (Invitrogen, Carlsbad, CA, USA). Reverse transcription was 
performed with a PrimeScript RT Reagent Kit (Takara, Tokyo, Japan). Real-time quantitative PCR assays were performed using Power SYBR Green PCR Master Mix and an ABI PRISM 7500 sequence detection system (Applied Biosystems, CA, USA). Glyceraldehyde-3-phosphate dehydrogenase (GAPDH) and U6 were used as internal standards for mRNA and microRNA, respectively. The primer sequences used in this study are shown in Table 2. Data were analyzed based on the $2^{-\Delta \Delta \mathrm{Ct}}$ method.

\section{Western blotting}

For total protein, cells were lysed in radioimmunoprecipitation assay (RIPA) buffer containing protease inhibitor mixture (Roche Applied Science, Mannheim, Germany). The lysates were harvested and centrifuged for $30 \mathrm{~min}$ at $12000 \mathrm{rpm}$ and $4{ }^{\circ} \mathrm{C}$. The Pierce BCA protein assay kit (Thermo Scientific) was utilized to detect the protein concentrations of the supernatants. Histone protein was extracted using the EpiQuik Total Histone Extraction Kit according to the manufacturer's protocol (Epigentek, USA). Aliquots $(20 \mu \mathrm{g})$ of the protein extracts were separated by $10 \%$ SDS PAGE and transferred to polyvinylidene difluoride membrane (Merck Millipore, Darmstadt, Germany). The membranes were blocked with $5 \%$ skim milk for $1 \mathrm{~h}$ and incubated with primary antibodies at $4{ }^{\circ} \mathrm{C}$ overnight. The ECL kit (CWBIO) was used to detect immunoreactive protein bands after incubation for $1 \mathrm{~h}$ with peroxidase-linked secondary antibodies at room temperature. The antibodies used were: anti-GAPDH, anti-PRMT3, anti-OCN (Abcam, Cambridge, UK), anti-H4, anti-RUNX2, anti-Flag (Cell Signaling, Danvers, MA, USA), and anti-H4R3me2a (Active Motif, Carlsbad, CA).

\section{Bone formation in vivo}

Beta-tricalcium phosphate ( $\beta$-TCP) (Bicon, Boston, MA, USA) scaffolds were utilized for the in vivo study. Cells were harvested when they reached $100 \%$ confluence, resuspended, and mixed with $\beta$-TCP for $1 \mathrm{~h}$. The mixtures were then implanted into the dorsal surface of nude mice subcutaneously. Each mouse held two implantation sites, which contained six groups of cells: hMSCs/NC, hMSCs/ PRMT3-sh1, hMSCs/PRMT3-sh2, hMSCs/Vector/ PRMT3sh, hMSCs/WT-PRMT3/PRMT3sh, and hMSCs/ Mut-PRMT3/PRMT3sh as described previously. The samples were harvested after 6-week implantation, fixed using $4 \%$ paraformaldehyde, and decalcified for 10 days in $10 \%$ EDTA (pH 7.4). The specimens were dehydrated and embedded in paraffin. Paraffin sections with $5-\mathrm{mm}$ thickness were stained with haematoxylin and eosin (H\&E) and Masson's trichrome stain. Osteogenic differentiation was validated by immunohistochemical (IHC) staining for Osteocalcin (OCN) (anti-OCN, Abcam, Cambridge, UK). All animal studies were performed under the approval of Experimental Animal Ethic Branch, Peking University Biomedical Ethic Committee.

\section{Virus infection and plasmid constructions}

Human and mice PRMT3 shRNA and the scramble negative control were purchased from GenePharma company (Suzhou, China). The shRNA target sequences for human PRMT3 were: PRMT3sh\#1, 5'-CAGCCTTGTAGCAGTGAGTGA-3' and PRMT3sh\#2, 5'CCTTGGGAGAAAGAAGAGTAT-3'; those for mouse Prmt3 were: Prmt3sh\#1, 5'-CCTCATTGTGACCCTGACTTT-3' and Prmt3sh\#2, 5'-CCTACGGTTGAATATATGAAT-3'. For gene overexpression, full length

Table 2 Sequences primers for real-time PCR or ChIP-qPCR

\begin{tabular}{lll}
\hline Genes & Forward primer & Reverse primer \\
\hline Primers for real-time PCR & & CGCCCAATACGACCAAATCCGT \\
GAPDH & TGTTCGACAGTCAGCCGCAT & ACTGCTTGCAGCCTTAAATGACTCT \\
RUNX2 & ACCACAAGTGCGGTGCAAAC & TCTTCCAGGTGTCAACGAGGT \\
ALP & TAAGGACATCGCCTACCAGCTC & CCTGCTTGGACACAAAGGCTGC \\
OCN & CACCATGAGAGCCCTCACACTC & AGTGTGTTTGGTGCTCTGAGGG \\
PRMT3 & GGACATCCATGTGCACGGCAA & TTCCAGTCAGACCCTTGGCAC \\
COL1A1 & CTGGCGCAGATGGTGTTGCT & AACGCTTCACGAATTGCGT \\
U6 & CTCGCTTCGGCAGCACA & AGCCGCGGGGATCGCCGAGGG \\
miR-3648 & GCGAGCACAGAATTAATACGAC & \\
Primers for ChIP-qPCR & & GTCGCACGAACGCCTGTC \\
Primer-1 & GTGGTCTCTCGTCTTCTCCC & GGGAGAAGACGAGAGACCAC \\
Primer-2 & GCCGATCCTCTTCTTCCCC &
\end{tabular}


PRMT3 and catalytic mutant PRMT3 were cloned into the $\mathrm{pLNB}$ vector with a mutant CBA promoter. The $\mathrm{pLNB}$ vector was resistant to blasticidin. Viral packaging was conducted as described previously ${ }^{38,63}$. Briefly, the pLNB vectors psPAX2 (Addgene) and pVSV-G (Clontech, USA) were co-transfected into HEK293T cells by using the PolyJet $^{\mathrm{tm}}$ Transfection Reagent (SignaGen Laboratories, Rockville, MD, USA) to produce lentiviruses. The supernatant was collected 36,48 , and $60 \mathrm{~h}$ after transfection, filtered, and centrifuged with PEG-it ${ }^{\text {tax }}$ (System Biosciences, SBI, Mountain View, CA, USA). The concentrated particles were titrated, aliquoted, and stored at $-80^{\circ} \mathrm{C}$ until further use. For lentivirus transfection, hMSCs was exposed to diluted viral supernatant with polybrene $(5 \mu \mathrm{g} / \mathrm{ml})$ for $24 \mathrm{~h}$. For construction of the PRMT3 knockdown cell line, puromycin was applied to choose the stably transfected cells after transfection for $72 \mathrm{~h}$. For construction of PRMT3 rescue cell line, PRMTsh cells were transfected with the vector, FlagPRMT3 wild-type (WT), and Flag-PRMT3 mutant (Mut). Puromycin and blasticidin were used to select the stably double-transfected cells after $72 \mathrm{~h}$ of transfection.

\section{ChIP and sequencing}

Briefly, cells were cross-linked with $1 \%$ formaldehyde, resuspended in lysis buffer (1\% SDS, $10 \mathrm{mM}$ EDTA, and $50 \mathrm{mM}$ Tris- $\mathrm{HCl}$ [pH 8.0]) on ice for $3 \mathrm{~min}$, and fragmented by sonication in RIPA ChIP buffer $(0.5 \mathrm{mM}$ EGTA, $140 \mathrm{mM} \mathrm{NaCl}, 10 \mathrm{mM}$ Tris-HCl, pH 7.5, $1 \%$ TritonX-100, $0.01 \%$ SDS, $1 \mathrm{mM}$ EDTA and protease inhibitor). The samples were then incubated overnight with Protein A/G-beads bound with antibodies [PRMT3 (Abcam, Cambridge, UK), H4R3me2a (Active Motif, Carlsbad, CA), H3 (Cell Signaling)]. After the crosslinking was reversed, immune complexes containing DNA were purified and eluted. The precipitated DNA for sequencing, including H4R3me2a and PRMT3, were constructed into libraries with NEBNext DNA Library Prep Reagent Set for Illumina (NEB) as described by the manufacturer.

\section{ChIP-seq data analysis}

Raw reads with adapters were trimmed and lowquality reads were discarded. We mapped the clean reads to a human reference genome (hg19) by using Burrows-Wheeler Aligner (BWA; ref1), retaining only unique non-duplicate reads. Fragment sizes were predicted by the following command: macs 2 predictd $-\mathrm{i}$ unique_non_duplicated_PRMT3.bed -f BED -g 3.1e9 -outdir./. Peaks were called by MACS (ref2) using input as controls by the following command: macs 2 callpeak $-t$ unique_non_duplicated_PRMT3.bed -c \$input.bed -g 3.1e9 -keep-dup all -nomodel -extsize \$predicted_fragment_size -q 0.01 .

\section{Transient transfection}

Cells were seeded at 70-80\% confluency and transfected with miR-3648 mimic, miR-3648 mimic control, miR3648 inhibitor, or miR-3648 inhibitor control (Ribobio, Guangzhou, China) at $100 \mathrm{nM}$ by using Lipofectamine 2000 (Invitrogen, Carlsbad, CA). The media was changed $6 \mathrm{~h}$ after transfection

\section{Cell proliferation assay}

The cell proliferation assay was performed with the Cell Counting Kit-8 (Dojindo, Kumamoto, Japan) according to the manufacturer's protocol. The media from each group was removed at the requisite time-point and cells were subsequently cultured in CCK-8-containing DMEM media for $2 \mathrm{~h}$ at $37^{\circ} \mathrm{C}$. Absorbance of each group was examined at $450 \mathrm{~nm}$ by using a microplate reader.

\section{Measurement of micro-computed tomography for bone samples}

Eight-week-old female C57BL/6 mice were provided by Vital River Corporation (Beijing, China) and maintained in pathogen-free facilities with a 12-h light/dark cycle. After 1 week, ovariectomy (OVX) or sham operation was bilaterally conducted by standard methods under general anesthesia with pentobarbital sodium injection $(50 \mathrm{mg} /$ $\mathrm{kg})^{64}$. The mice were sacrificed by $\mathrm{CO}_{2}$ asphyxiation at 6 or 12 weeks. The femurs were harvested and analyzed by highresolution microtomography (Inveon, Siemens, Munich, Germany). Images were obtained at an effective pixel size of $8.82 \mu \mathrm{m}$, voltage of $80 \mathrm{kV}$, tube current of $500 \mu \mathrm{A}$, and exposure time of $1500 \mathrm{~ms}$ in each of the 360 rotational steps. Parameters were analyzed by Inveon Research Workplace (Siemens), and included BV/TV, Tb.Th, and Tb. $\mathrm{N}$ according to the guidelines set by the American Society for Bone and Mineral Research (ASBMR).

\section{Mini-osmotic pump (ALZET) implantation}

Mice were randomly assigned to six groups of five animals each. The groups were (1) 2 week/CTR, (2) 2 week/ SGC707, (3) 4 week/CTR, (4) 4 week/SGC707, (5) 6 week/ CTR, and (6) 6 week/SGC707. After the mice were anesthetized with pentobarbital sodium injection $(50 \mathrm{mg} / \mathrm{kg})$, the backs were subcutaneously implanted with an Alzet mini-pump (model 2002, Alzet, Cupertino, CA, USA). The Alzet pumps were used with a delivery rate at $0.5 \mu \mathrm{L} / \mathrm{h}$, or $200 \mu \mathrm{L}$ over 2 weeks. To ensure continuous exposure during the 4- or 6-week period, two or three mini-osmotic pumps were consecutively implanted. Alzet pumps were filled according to the manufacturer's instructions. To achieve a mean targeted concentration of $20 \mathrm{mg} / \mathrm{kg} /$ day, SGC707 was dissolved in DMSO at a concentration of $28 \mathrm{mg} / \mathrm{ml}$. After ALZET implantation for 2, 4, or 6 weeks, the mice were euthanized and the femurs were harvested. The femurs were fixed in $4 \%$ paraformaldehyde for $24 \mathrm{~h}$ 
and scanned by micro-CT. Then the femurs were embedded in paraffin and sectioned to conduct $\mathrm{H} \& \mathrm{E}$ staining and IHC staining for PRMT3 (Abcam, Cambridge, UK), RUNX2 (Cell Signaling, Danvers, MA, USA), and OCN (Abcam, Cambridge, UK).

\section{Statistical analyses}

The statistical analyses were done with SPSS Statistics 20.0 software (IBM, Armonk, NY, USA). Comparisons between two groups were conducted by Student's $t$-tests, and multiple comparisons were performed by one-way analysis of variance (ANOVA) followed by a Tukey's post hoc test. Data were shown as the mean \pm standard deviation (SD) of three assays per group. $P$-values $<0.05$ were considered statistically significant.

\section{Accession number}

All of the ChIP-seq data were deposited in GEO under the accession number GSE124674. To review GEO accession GSE124674: Go to Enter token adurqkkihfcfbkb into the box.

\section{Acknowledgements}

This project was supported by grants from the National Natural Science Foundation of China (81500822 to P.Z. and 81570953 to Y.Z), the Project for Culturing Leading Talents in Scientific and Technological Innovation of Beijing (Z171100001117169), the PKU School of Stomatology for Talented Young Investigators (PKUSS20140109), the Beijing Nova Program

\section{(Z181100006218037)}

\section{Author details}

${ }^{1}$ Department of Prosthodontics, Peking University School and Hospital of Stomatology, 100081 Beijing, China. ${ }^{2}$ National Engineering Lab for Digital and Material Technology of Stomatology, National Clinical Research Center for Oral Diseases, Peking University School and Hospital of Stomatology, 100081 Beijing, China. ${ }^{3}$ Beijing Advanced Innovation center for Genomics (ICG), College of Life Sciences, Peking University, 100871 Beijing, China

\section{Conflict of interest}

The authors declare that they have no conflict of interest.

\section{Publisher's note}

Springer Nature remains neutral with regard to jurisdictional claims in published maps and institutional affiliations.

Supplementary Information accompanies this paper at (https://doi.org/ 10.1038/s41419-019-1815-7).

Received: 6 April 2019 Revised: 3 July 2019 Accepted: 12 July 2019 Published online: 05 August 2019

\section{References}

1. Pensak, M. J. \& Lieberman, J. R. Gene therapy for bone regeneration. Curr. Pharm. Des. 19, 3466-3473 (2013).

2. Seeman, E. \& Delmas, P. D. Bone quality-the material and structural basis of bone strength and fragility. N. Engl. J. Med. 354, 2250-2261 (2006).

3. Bianco, P. et al. The meaning, the sense and the significance: translating the science of mesenchymal stem cells into medicine. Nat. Med. 19, 35-42 (2013).

4. Pacifici, R. Estrogen deficiency, T cells and bone loss. Cell Immunol. 252, 68-80 (2008).
5. Gibon, E., Lu, L. \& Goodman, S. B. Aging, inflammation, stem cells, and bone healing. Stem Cell Res. Ther. 7, 44 (2016).

6. Lee, W. C., Guntur, A. R., Long, F. \& Rosen, C. J. Energy Metabolism of the Osteoblast: Implications for Osteoporosis. Endocr. Rev. 38, 255-266 (2017).

7. Caplan, A. I. Adult mesenchymal stem cells for tissue engineering versus regenerative medicine. J. Cell Physiol. 213, 341-347 (2007).

8. Sponer, P., Kucera, T., Diaz-Garcia, D. \& Filip, S. The role of mesenchymal stem cells in bone repair and regeneration. Eur. J. Orthop. Surg. Trauma. 24, 257-262 (2014).

9. Fitzsimmons, R., Mazurek, M. S., Soos, A. \& Simmons, C. A. Mesenchymal stromal/stem cells in regenerative medicine and tissue engineering. Stem Cells Int. 2018, 8031718 (2018).

10. Liu, L. et al. Arginine methylation of SREBP1a via PRMT5 promotes de novo lipogenesis and tumor growth. Cancer Res. 76, 1260-1272 (2016).

11. Blanc, R. S. \& Richard, S. Arginine methylation: the coming of age. Mol. Cell 65, 8-24 (2017).

12. Galligan, J. J. et al. Methylglyoxal-derived posttranslational arginine modifications are abundant histone marks. Proc. Natl Acad. Sci. USA 115, 9228-9233 (2018).

13. Vadnais, C. et al. GFI1 facilitates efficient DNA repair by regulating PRMT1 dependent methylation of MRE11 and 53BP1. Nat. Commun. 9, 1418 (2018).

14. Kawabe, Y., Wang, Y. X., McKinnell, I. W., Bedford, M. T. \& Rudnicki, M. A. Carm1 regulates $\operatorname{Pax} 7$ transcriptional activity through $M L L 1 / 2$ recruitment during asymmetric satellite stem cell divisions. Cell Stem Cell 11, 333-345 (2012).

15. Tsai, W. W. et al. PRMT5 modulates the metabolic response to fasting signals. Proc. Natl Acad. Sci. USA 110, 8870-8875 (2013).

16. Park, M. J. et al. Thioredoxin-interacting protein mediates hepatic lipogenesis and inflammation via PRMT1 and PGC-1alpha regulation in vitro and in vivo. J. Hepatol. 61, 1151-1157 (2014).

17. Infantino, S. et al. Arginine methylation catalyzed by PRMT1 is required for B cell activation and differentiation. Nat. Commun. 8, 891 (2017).

18. Hamard, P. J. et al. PRMT5 regulates DNA repair by controlling the alternative splicing of histone-modifying enzymes. Cell Rep. 24, 2643-2657 (2018).

19. Gou, Y. et al. Prmt1 regulates craniofacial bone formation upstream of Msx1. Mech. Dev. 152, 13-20 (2018).

20. Choi, J. H. et al. PRMT1 mediates RANKL-induced osteoclastogenesis and contributes to bone loss in ovariectomized mice. Exp. Mol. Med 50, 111 (2018).

21. Dong, Y. et al. Inhibition of PRMT5 suppresses osteoclast differentiation and partially protects against ovariectomy-induced bone loss through downregulation of CXCL10 and RSAD2. Cell Signal 34, 55-65 (2017).

22. Kota, S. K., Roening, C., Patel, N., Kota, S. B. \& Baron, R. PRMT5 inhibition promotes osteogenic differentiation of mesenchymal stromal cells and represses basal interferon stimulated gene expression. Bone 117, 37-46 (2018).

23. Tang, J., Gary, J. D., Clarke, S. \& Herschman, H. R. PRMT 3, a type I protein arginine $\mathrm{N}$-methyltransferase that differs from PRMT1 in its oligomerization, subcellular localization, substrate specificity, and regulation. J. Biol. Chem. 273, 16935-16945 (1998).

24. Frankel, A. \& Clarke, S. PRMT3 is a distinct member of the protein arginine Nmethyltransferase family. Conferral of substrate specificity by a zinc-finger domain. J. Biol. Chem. 275, 32974-32982 (2000).

25. Bachand, F. \& Silver, P. A. PRMT3 is a ribosomal protein methyltransferase that affects the cellular levels of ribosomal subunits. EMBO J. 23, 2641-2650 (2004).

26. Swiercz, R., Person, M. D. \& Bedford, M. T. Ribosomal protein S2 is a substrate for mammalian PRMT3 (protein arginine methyltransferase 3). Biochem J. $\mathbf{3 8 6}$, 85-91 (2005).

27. Swiercz, R., Cheng, D., Kim, D. \& Bedford, M. T. Ribosomal protein rpS2 is hypomethylated in PRMT3-deficient mice. J. Biol. Chem. 282, 16917-16923 (2007).

28. Miyata, S., Mori, Y. \& Tohyama, M. PRMT3 is essential for dendritic spine maturation in rat hippocampal neurons. Brain Res. 1352, 11-20 (2010).

29. Kim, D. I. et al. PRMT3 regulates hepatic lipogenesis through direct interaction with LXRalpha. Diabetes 64, 60-71 (2015).

30. Brais, B. et al. Short GCG expansions in the PABP2 gene cause oculopharyngeal muscular dystrophy. Nat. Genet 18, 164-167 (1998).

31. Tavanez, J. P. et al. Hsp70 chaperones and type I PRMTs are sequestered at intranuclear inclusions caused by polyalanine expansions in PABPN1. PLOS ONE 4, e6418 (2009).

32. Singh, $\mathrm{V}$. et al. DAL-1/4.1B tumor suppressor interacts with protein arginine $\mathrm{N}$ methyltransferase 3 (PRMT3) and inhibits its ability to methylate substrates in vitro and in vivo. Oncogene 23, 7761-7771 (2004). 
33. Heller, G. et al. Downregulation of TSLC1 and DAL-1 expression occurs frequently in breast cancer. Breast Cancer Res. Treat. 103, 283-291 (2007)

34. Chen, $X$. et al. Expression of nitric oxide related enzymes in coronary heart disease. Basic Res. Cardiol. 101, 346-353 (2006).

35. Kaniskan, H. U. et al. A potent, selective and cell-active allosteric inhibitor of protein arginine methyltransferase 3 (PRMT3). Angew. Chem. Int Ed. Engl. 54, 5166-5170 (2015).

36. Khosla, S., Westendorf, J. J. \& Oursler, M. J. Building bone to reverse osteoporosis and repair fractures. J. Clin. Invest 118, 421-428 (2008).

37. Khosla, S. Pathogenesis of osteoporosis. Transl. Endocrinol. Metab. 1, 55-86 (2010).

38. Zhang, P. et al. Histone H3K9 acetyltransferase PCAF is essential for osteogenic differentiation through bone morphogenetic protein signaling and may be involved in osteoporosis. Stem Cells 34, 2332-2341 (2016).

39. Allis, C. D. \& Jenuwein, T. The molecular hallmarks of epigenetic control. Nat. Rev. Genet 17, 487-500 (2016).

40. Egger, G., Liang, G., Aparicio, A. \& Jones, P. A. Epigenetics in human disease and prospects for epigenetic therapy. Nature 429, 457-463 (2004).

41. Ahuja, N., Sharma, A. R. \& Baylin, S. B. Epigenetic therapeutics: a new weapon in the war against cancer. Annu Rev. Med 67, 73-89 (2016).

42. Ermolaeva, M., Neri, F., Ori, A. \& Rudolph, K. L. Cellular and epigenetic drivers of stem cell ageing. Nat. Rev. Mol. Cell Biol. 19, 594-610 (2018).

43. Ng, S. S., Yue, W. W., Oppermann, U. \& Klose, R. J. Dynamic protein methylation in chromatin biology. Cell Mol. Life Sci. 66, 407-422 (2009).

44. Bedford, M. T. \& Clarke, S. G. Protein arginine methylation in mammals: who, what, and why. Mol. Cell 33, 1-13 (2009).

45. Gordon, J., Stein, J. L., Westendorf, J. J. \& van Wijnen, A. J. Chromatin modifiers and histone modifications in bone formation, regeneration, and therapeutic intervention for bone-related disease. Bone 81, 739-745 (2015).

46. Wei, Y. et al. CDK1-dependent phosphorylation of EZH2 suppresses methylation of $\mathrm{H} 3 \mathrm{~K} 27$ and promotes osteogenic differentiation of human mesenchymal stem cells. Nat. Cell Biol. 13, 87-94 (2011).

47. Ge, W. et al. The epigenetic promotion of osteogenic differentiation of human adipose-derived stem cells by the genetic and chemical blockade of histone demethylase LSD1. Biomaterials 35, 6015-6025 (2014).

48. Siarheyeva, A. et al. An allosteric inhibitor of protein arginine methyltransferase 3. Structure 20, 1425-1435 (2012).

49. Nahon, J. E., Groeneveldt, C., Geerling, J. J., van Eck, M. \& Hoekstra, M. Inhibition of protein arginine methyltransferase 3 activity selectively impairs liver $X$ receptor-driven transcription of hepatic lipogenic genes in vivo. $\mathrm{Br}$. J. Pharm. 175, 3175-3183 (2018).
50. Guo, H., Ingolia, N. T., Weissman, J. S. \& Bartel, D. P. Mammalian microRNAs predominantly act to decrease target mRNA levels. Nature 466, 835-840 (2010).

51. Eskildsen, T. et al. MicroRNA-138 regulates osteogenic differentiation of human stromal (mesenchymal) stem cells in vivo. Proc. Natl Acad. Sci. USA 108, 6139-6144 (2011)

52. Mei, Y. et al. miR-21 modulates the ERK-MAPK signaling pathway by regulating SPRY2 expression during human mesenchymal stem cell differentiation. J. Cell Biochem. 114, 1374-1384 (2013).

53. Chen, S., Zheng, Y., Zhang, S., Jia, L. \& Zhou, Y. Promotion effects of miR-375 on the osteogenic differentiation of human adipose-derived mesenchymal stem cells. Stem Cell Rep. 8, 773-786 (2017).

54. Mallappa, C. et al. The expression of myogenic microRNAs indirectly requires protein arginine methyltransferase (Prmt)5 but directly requires Prmt4. Nucleic Acids Res. 39, 1243-1255 (2011).

55. Lee, S. H. et al. A feedback loop comprising PRMT7 and miR-24-2 interplays with Oct4, Nanog, Klf4 and c-Myc to regulate stemness. Nucleic Acids Res. 44, 10603-10618 (2016)

56. Wang, $\mathrm{H}$. et al. Methylation of histone $\mathrm{H} 4$ at arginine 3 facilitating transcriptional activation by nuclear hormone receptor. Science 293, 853-857 (2001).

57. Strahl, B. D. et al. Methylation of histone $\mathrm{H} 4$ at arginine 3 occurs in vivo and is mediated by the nuclear receptor coactivator PRMT1. Curr. Biol. 11, 996-1000 (2001).

58. Sen, S. et al. PRMT1 plays a critical role in Th17 differentiation by regulating reciprocal recruitment of STAT3 and STAT5. J. Immunol. 201, 440-450 (2018).

59. Wagner, S., Weber, S., Kleinschmidt, M. A., Nagata, K. \& Bauer, U. M. SETmediated promoter hypoacetylation is a prerequisite for coactivation of the estrogen-responsive pS2 gene by PRMT1. J. Biol. Chem. 281, 27242-27250 (2006).

60. Di Lorenzo, A. \& Bedford, M. T. Histone arginine methylation. FEBS Lett. 585 2024-2031 (2011)

61. Rashid, F., Awan, H., Shah, A., Chen, L. \& Shan, G. Induction of miR-3648 upon ER stress and its regulatory role in cell proliferation. Int. J. Mol. Sci. 18, 1375 (2017).

62. Scimeca, J. C. \& Verron, E. The multiple therapeutic applications of miRNAs for bone regenerative medicine. Drug Disco. Today 22, 1084-1091 (2017).

63. Zhang, M. et al. RSPO3-LGR4 regulates osteogenic differentiation of human adipose-derived stem cells via ERK/FGF signalling. Sci. Rep. 7, 42841 (2017).

64. Jin, $C$. et al. Inhibition of SLC7A11 by sulfasalazine enhances osteogenic differentiation of mesenchymal stem cells by modulating BMP2/4 expression and suppresses bone loss in ovariectomized mice. J. Bone Min. Res. 32 508-521 (2017) 